\title{
Paradigm-changing osimertinib approval in front-line for advanced NSCLC
}

T he US Food and Drug Administration awarded regulatory approval this spring to the third-generation epidermal growth factor receptor (EGFR) inhibitor osimertinib for the treatment of patients with exon 19 deletion- or exon21 L858R mutation-positive advanced non-small-cell lung cancer (NSCLC) not previously treated for advanced disease.

Osimertinib is designed to target both sensitizing and resistant mutant forms of EGFR, but not the wildtype protein, in an effort to improve safety and efficacy compared with other standard of care (SoC) EGFR inhibitors. It was previously approved in the second-line setting in NSCLC following failure of prior EGFR inhibitor therapy in 2015. The current approval represents a paradigm shift in the front-line treatment of advanced NSCLC, reinforcing the role of osimertinib, which has been recommended in this setting by the National Comprehensive Cancer Network Guidelines in Oncology for more than a year.

Approval was based on the phase 3, multicenter, international, randomized, double-blind, active-controlled FLAURA trial. A total of 556 patients were randomized 1:1 to receive an oral daily dose of $80 \mathrm{mg}$ osimertinib or gefitinib $250 \mathrm{mg}$ or erlotinib $150 \mathrm{mg}$. The trial was conducted during December 2014 through March 2016 at 132 sites in 29 countries.

Eligible patients were aged 18 or over and had locally advanced or metastatic NSCLC, had not previously received treatment for advanced disease, were eligible for first-line treatment with erlotinib or gefitinib, had locally or centrally confirmed EGFR exon 19 deletion or L858R mutations alone or concurrently with other EGFR mutations, and a World Health Organization Performance Status of 0 (fully active, able to carry on all predisease performance without restriction) or 1 (restricted in strenuous activity but ambulatory and able to carry out light work), and a minimum life expectancy of 12 weeks.

Patients with central nervous system metastases were eligible if their condition was neurologically stable. Patients who had previous definitive treatment or glucocorticoid therapy had to have completed it at least 2 weeks before the start of the trial. Patients were excluded from the trial if they had any previous treatment with any systemic anticancer therapy for advanced NSCLC, had major surgery within 4 weeks of the first dose of the study drug, had radi-

\section{What's new, what's important}

Safety and encouraging improvement in PFS are the hallmarks of the approval of the EGFR inhibitor osimertinib for previously untreated advanced NSCLC. The approval was based on the FLAURA trial in which 556 patients were randomized to receive $80 \mathrm{mg}$ osimertinib PO or gefitinib $250 \mathrm{mg}$ or erlotinib $150 \mathrm{mg}$.

Osimertinib cut the risk of progression or death by more than $50 \%$ compared with standard TKI therapy. The estimated median PFS was 18.9 months with osimertinib (10.2 months for erlotinib/gefitinib). There was a PFS benefit across all prespecified subgroups, including patients with CNS metastases (15.2 months vs 9.6 months). Confirmed ORR was $77 \%$ and $69 \%$, and estimated DoR was 17.6 months and 9.6 months. At the time of analysis, there were too few deaths to compare OS. The most common AEs were diarrhea, rash, dry skin, nail toxicity, stomatitis, and reduced appetite.

Prescribing information warns about ILD and pneumonitis, QTc interval prolongation, cardiomyopathy, keratitis, and embryofetal toxicity. Treatment should be withheld if patients present with respiratory symptoms indicative of ILD and discontinued on confirmation of ILD. Treatment should be permanently discontinued with QTc interval prolongation with signs and symptoms of lifethreatening arrhythmia. Cardiac monitoring, including assessment of LVEF, should be done at baseline and during treatment in patients with cardiac risk factors. Patients with signs and symptoms of keratitis should see an ophthalmologist.

- Jame Abraham, MD, FACP (abrahaj5@ccf.org)

ation therapy to more than $30 \%$ of the bone marrow or a wide field of radiation within 4 weeks of the first dose of the study drug, or were currently receiving potent inhibitors or inducers of cytochrome P450 3A4.

Osimertinib cut the risk of disease progression or death by more than $50 \%$ compared with standard TKI therapy. The estimated median progression-free survival (PFS) was 18.9 months with osimertinib, compared with 10.2 months for erlotinib or gefitinib (hazard ratio [HR]: 0.46; $P<$ $.0001)$. PFS benefit extended across all prespecified subgroups, including patients with CNS metastases (median PFS: 15.2 months vs 9.6 months; HR: $0.47 ; P=.0009$ ). Confirmed overall response rate was $77 \%$ and $69 \%$ in the study and SoC groups, respectively, and estimated duration 


\section{Mechanism of action: EGFR inhibitors}

The next generation. The epidermal growth factor receptor (EGFR) gene encodes a tyrosine kinase receptor protein that activates key intracellular signaling pathways involved in cell survival, proliferation, and other vital cellular processes. These pathways are often corrupted in cancer cells - commonly through activating mutations in the EGFR gene - to facilitate the transformation of a normal cell into a malignant one.

EGFR mutations are particularly common in non-small-cell lung cancers (NSCLCs) and represent one of the major drivers of this cancer type. This has served as the impetus for the development of small molecule inhibitors of the EGFR designed to block the activity of the most common mutant forms of the protein - those encoded by an EGFR gene with exon 19 deletions and exon 21 L858R point mutations (known as "sensitizing" mutations).

The first generation of EGFR inhibitors, which included erlotinib and gefitinib, revolutionized the treatment of patients with EGFRmutant NSCLC. However, within a year, most patients who initially respond to these drugs typically develop acquired resistance that drives treatment failure.

Investigators have identified the most common mechanisms of resistance, which include the T790M mutation in more than half of cases. Known as a "gatekeeper mutation," it affects an amino acid residue that controls access to a hydrophobic pocket within the active site of the kinase, blocking the ability of the inhibitors to bind to their target, as well as altering the affinity of the mutant EGFR for adenosine triphosphate (ATP) and thus reducing the potency of ATP-competitive inhibitors.

A second generation of EGFR inhibitors was developed and designed to have activity against the T790M mutant form of the EGFR. These inhibitors also showed promise, but have been limited by the toxicity that results from their inhibition of the wildtype form of the EGFR.

Osimertinib represents one of the third generation of drugs that is designed not only to target both sensitizing and mutant forms of the EGFR, but to have limited efficacy against the wild-type form of the protein, in the hopes of improving efficacy and tolerability. Osimertinib has a distinct structure and pharmacology that render it 200-fold more selective for mutant forms over wild-type EGFR.

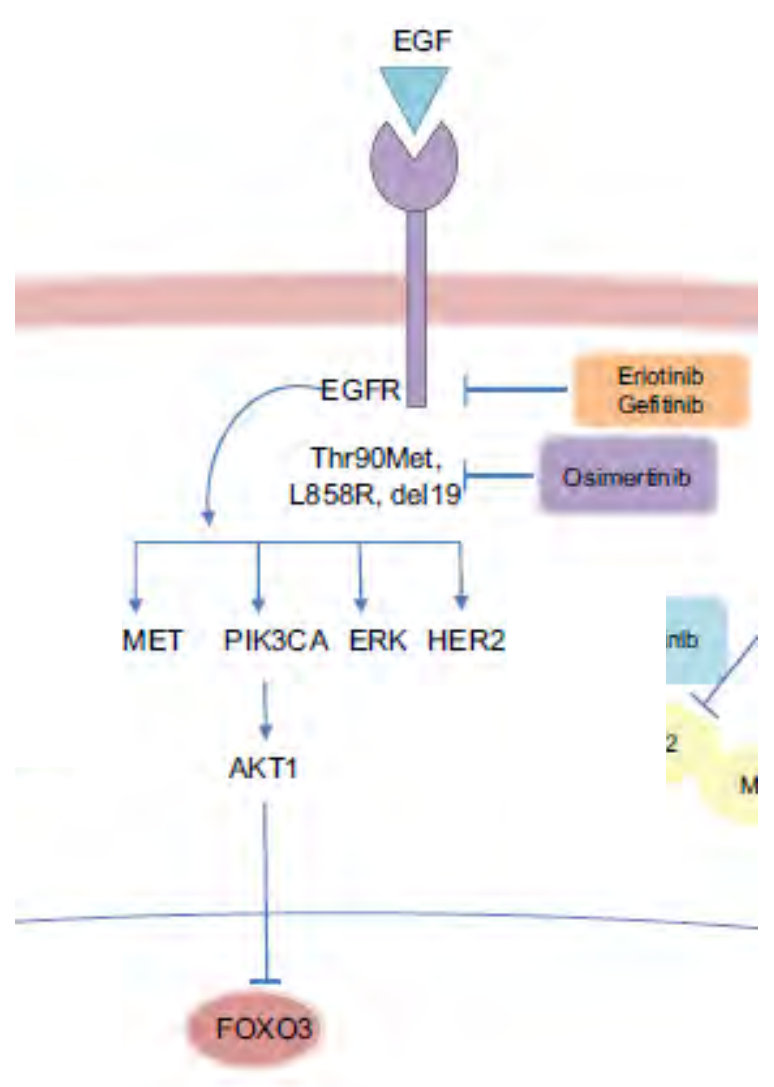

Mutations in the EGFR gene are central drivers of non-small cell lung cancer (NSCLC), which has served as the rationale for the development of EGFR-targeted tyrosine kinase inhibitors, such as erlotinib and gefitinib. Unfortunately, most patients rapidly develop resistance, most commonly due to additional EGFR mutations. Next-generation TKIs like osimertinib are able to target both sensitizing and resistant EGFR mutations. Recreated under a Creative Commons Attribution 4.0 International License. Denisenko TV et al. Cell deathbased treatment of lung adenocarcinoma. Cell Death Dis. 2018;9:117. of response (DoR) was 17.6 months and 9.6 months. At the time of analysis, there were too few deaths to compare overall survival.

The most common adverse events (AEs) experienced by patients treated with osimertinib were diarrhea, rash, dry skin, nail toxicity, stomatitis, and reduced appetite. Serious AEs occurred in $4 \%$ of patients treated with osimertinib, most commonly involving pneumonia, interstitial lung disease/pneumonitis, and pulmonary embolism (PE). The rate of grade $3 / 4$ AEs was $33.7 \%$ in the osimertinib group and $44.8 \%$ in the $\mathrm{SoC}$ group. Patients treated with osimer- tinib were less likely to discontinue treatment due to AEs ( $13.3 \%$ vs $18.1 \%$ of those receiving SoC).

Osimertinib is marketed as Tagrisso by AstraZeneca and the recommended dose is $80 \mathrm{mg}$ orally once daily, with or without food. The prescribing information details warnings and precautions relating to interstitial lung disease and pneumonitis, QTc interval prolongation, cardiomyopathy, keratitis, and embryofetal toxicity.

Treatment with osimertinib should be withheld in patients presenting with worsening of respiratory symptoms indicative of ILD and permanently discontinued if 
ILD is confirmed. Electrocardiograms and electrolytes should be monitored periodically in patients with congenital long QTc syndrome, congestive heart failure, electrolyte abnormalities or in patients taking medications known to prolong QTc interval. Treatment should be permanently discontinued in those who develop QTc interval prolongation with signs and symptoms of life-threatening arrhythmia.

Cardiac monitoring, including assessment of left ven-

\section{References}

1. US Food and Drug Administration Website. FDA approves osimertinib for first-line treatment of metastatic NSCLC with most common EGFR mutations. https://www.fda.gov/drugs/informationondrugs/approveddrugs/ucm605113.htm. Last updated April 18, 2018. Accessed October 6, 2018.

2. Soria J-C, Ohe Y, Vansteenkiste J, et al. Osimertinib in untreated tricular ejection fraction should be performed at baseline and throughout treatment in patients with cardiac risk factors and treatment should be permanently discontinued in patients who develop symptomatic congestive heart failure. Patients with signs and symptoms of keratitis should be referred to an ophthalmologist. Osimertinib can cause fetal harm and patients should be advised of the potential risk and the need for effective contraception use during treatment and for 6 weeks after the final dose is administered.

EGFR-mutated advanced non-small-cell lung cancer. N Engl J Med. 2018;378:113-125.

3. Tagrisso (osimertinib tablets) for oral use. Prescribing information. AstraZeneca. https://www.azpicentral.com/tagrisso/tagrisso. pdf\#page=1. August 2018. Accessed October 6, 2018. 